\title{
Trivium
}

Revue franco-allemande de sciences humaines et sociales - Deutsch-französische Zeitschrift für Geistesund Sozialwissenschaften

$31 \mid 2020$

La culture politique de la République romaine

\section{L'historiographie française de la République romaine : six décennies de recherche (1960-2020)}

Jean-Michel David et Frédéric Hurlet

\section{OpenEdition \\ Journals}

Édition électronique

URL : https://journals.openedition.org/trivium/7248

DOI : $10.4000 /$ trivium.7248

ISBN : 1963-1820

ISSN : 1963-1820

Éditeur

Les éditions de la Maison des sciences de l'Homme

Référence électronique

Jean-Michel David et Frédéric Hurlet, «L'historiographie française de la République romaine : six décennies de recherche (1960-2020) », Trivium [En ligne], 31 | 2020, mis en ligne le 30 juin 2020, consulté le 24 août 2021. URL : http://journals.openedition.org/trivium/7248 ; DOI : https://doi.org/ $10.4000 /$ trivium. 7248

Ce document a été généré automatiquement le 24 août 2021.

\section{(c) $(1) \Theta \Theta$}

Les contenus des la revue Trivium sont mis à disposition selon les termes de la Licence Creative Commons Attribution - Pas d'Utilisation Commerciale - Pas de Modification 4.0 International. 


\title{
L'historiographie française de la République romaine : six décennies de recherche (1960-2020)
}

\author{
Jean-Michel David et Frédéric Hurlet
}

1 Dresser un état des lieux actuel de la recherche en France sur la République romaine revient à parler non seulement d'individualités qui incarnent aujourd'hui une historiographie nationale, mais aussi de ceux qui ont été nos maîtres ou les maitres de nos maîtres. Pour la France, une figure se détache, celle de Claude Nicolet (1930-2010), qui a littéralement fait école à partir des années $60 \mathrm{du} \mathrm{XX}^{\mathrm{e}}$ siècle et jusque dans les années $1990^{1}$. Mais il n'était pas isolé et nous commencerons par relever quelques autres tendances contemporaines.

\section{La richesse et la diversité de l'historiographie française sur la République}

2 La première tendance tint à la vitalité de l'Histoire économique. Ce fut surtout Jean Andreau ${ }^{2}$ qui, dans ce domaine, affronta avec le plus de détermination les questions théoriques. On peut distinguer deux parties principales dans ses publications : d'une part des recherches sur la banque, les banquiers, les milieux d'affaires et les pratiques financières; d'autre part des études plus générales sur l'économie antique, où il entrait dans le débat qui portait sur son caractère moderne ou primitif, c'est-à-dire sur sa capacité ou non à se développer. On peut rappeler ici les travaux d'Yves Roman sur la circulation et les échanges en Gaule au I ${ }^{\mathrm{er}}$ siècle avant notre ère, qu'il poursuivit avec Danièle Roman par une histoire de la Gaule et un ouvrage synthétique paru en 2016 sur le processus d'intégration de l'Empire.

3 La seconde fut le fait de Pierre Lévêque et de Monique Clavel-Lévêque, qui créèrent à Besançon un centre de recherche consacré principalement à l'étude de l'esclavage et des conditions de production agraire, en particulier des cadastres des cités antiques. Les résultats de ces enquêtes étaient importants aussi bien à propos de la place des 
esclaves que des pratiques de fondation des colonies en Gaule et en Italie. On peut ainsi citer les travaux de Monique Clavel-Lévêque, François Favory, Gérard Chouquer et Antonio Gonzales's, qui d'une certaine façon firent école, puisque la méthode fut étendue à d'autres régions méditerranéennes.

La troisième, liée à l'importance des analyses d'anthropologie culturelle qui se développèrent en France dans les années 60 à 90, sous l'influence de Jean-Pierre Vernant et de Pierre Vidal-Naquet, fut le renouvellement de l'étude de la religion romaine qui s'affirma dans l'œuvre de John Scheid. Comme chacun sait, John Scheid a établi que la religion romaine était fondamentalement une religion de la pratique qui définissait une orthopraxie dans le cadre de laquelle les relations des hommes avec les dieux étaient gérées de façon en quelque sorte contractuelle ${ }^{4}$. Les ouvrages français précédents avaient développé un autre type d'analyse où la religion apparaissait davantage comme une affaire de sentiment et d'adhésion personnelle ${ }^{5}$.

Il faut également signaler les travaux des juristes. Si l'enseignement du droit romain dans les universités françaises n'a pas conservé la même importance qu'en Italie ou en Allemagne, il y a tout de même eu de grands noms qui ressortent en France, en particulier au siècle passé celui de André Magdelain (1917-1993), qui a publié de nombreux travaux utiles aux historiens de la République romaine ${ }^{6}$. Actuellement, c'est le nom de Michel Humbert qui s'impose dans ce secteur et pour cette période : auteur d'un livre sur le processus de municipalisation et les conditions de la citoyenneté en Italie jusqu'à la guerre dite sociale qui constitua un apport définitif, il a publié récemment, en 2018, une nouvelle édition de référence de la loi des XII Tables ${ }^{7}$. Un autre juriste dont les travaux sur la République romaine se sont révélés d'une grande fécondité pour les historiens sont ceux de Yan Thomas, qui a du reste travaillé avec Nicolet notamment dans le cadre de la réédition en 1984 de la traduction française du Droit public romain de Th. Mommsen ${ }^{8}$.

Un dernier courant de recherche à mentionner dans ce cadre est celui qui adopte une perspective provinciale et qui est le dénominateur commun des travaux menés à Bordeaux au sein de l'ancien centre Pierre Paris, fondé par Robert Étienne et devenu l'Institut Ausonius. Même si le centre d'intérêt principal reste l'époque impériale, il faut signaler les travaux sur l'Hispanie romaine à l'époque républicaine publiés par plusieurs collègues : Jean-Michel Roddaz, dont une part de la production scientifique a porté aussi sur l'époque triumvirale et augustéenne ${ }^{9}$; Nathalie Barrandon, qui a étudié dans sa thèse les mutations des sociétés indigènes d'Hispanie centrale et septentrionale de 133 à 27 av. J.-C. et poursuivi ses recherches en organisant avec François Kirbihler deux colloques sur le gouvernement des provinces à l'époque républicaine et en publiant récemment sur le thème des massacres de la République romaine un livre issu de son Habilitation ${ }^{10}$; François Cadiou, qui a consacré sa thèse aux armées romaines de la péninsule Ibérique de la seconde guerre punique à la bataille de Munda ${ }^{11}$.

Nous évoquerons enfin d'un mot l'œuvre de Paul Veyne, contemporaine de celle de Claude Nicolet ${ }^{12}$. La plupart de ses études et publications portaient sur le monde romain en général et traitaient de sujets plus récents que la République romaine. Mais la démarche intellectuelle qui était la sienne, faite d'interrogation critique, d'analyses sociologiques et souvent aussi de propositions paradoxales, suscitait l'intérêt et encourageait à rompre avec la révérence obligée à l'égard de l'Antiquité.

Un signe de la vitalité de l'historiographie française sur la République romaine tient à la forte influence qu'elle a exercée sur l'historiographie de langue française de 
Belgique. La division de ce pays entre deux communautés linguistiques et culturelles, celle des Francophones et celle des Néerlandophones, s'est en la matière accentuée ces dernières décennies et a eu pour conséquence que les historiens néerlandophones de Rome publient désormais en anglais, se rapprochant ainsi naturellement des réseaux anglophones, alors qu'ils considéraient auparavant le français comme la principale langue de communication ${ }^{13}$. Les Francophones ont en revanche continué à se tourner vers la France, par attraction naturelle. Le tableau de l'historiographie francophone de Belgique est nécessairement complexe dans un pays éclaté où chaque université a été et est toujours fière de son autonomie. L'Université de Liège a été dominée par une forte tradition philologique, dont le plus beau fruit est l'étude du bilinguisme à Rome et qui a débouché sur deux ouvrages consacrés à deux historiens grecs majeurs et à leur manière de traduire en grec des réalités institutionnelles romaines d'époque républicaine : celui de Michel Dubuisson tout d'abord, intitulé Le latin de Polybe ${ }^{14}$; celui d'Étienne Famerie ensuite, portant sur le lexique d'Appien ${ }^{15}$. Pour l'Université de Louvain, le nom qui ressort est celui de Françoise Van Haeperen, qui est une spécialiste reconnue de la religion romaine ${ }^{16}$. Pour finir, on mentionnera pour l'Université de Namur le nom de Pierre Assenmaker, qui intègre à l'analyse les sources numismatiques en les combinant avec les sources épigraphiques et littéraires et dont la thèse, publiée en 2014, était consacrée au développement et aux manifestations de l'idéologie impératoriale à l'époque de Marius et Sylla ${ }^{17}$.

\section{Le moment Nicolet}

9 Participant à la richesse de l'historiographie française et francophone et à sa diversité, les travaux de Claude Nicolet constituèrent la principale source de renouvellement de la réflexion sur la République romaine. Auteur d'une thèse d'État sur l'ordre équestre à l'époque républicaine ${ }^{18}$, il a prolongé ce travail académique par des ouvrages sur la République romaine qui ont fait date non seulement en France, mais aussi à un niveau international: en particulier Le métier de citoyen (1976); on citera aussi Rome et la conquête du monde méditerranéen, ouvrage pour lequel il a pris seul en charge la rédaction du premier tome sur les structures de l'Italie romaine (1977) et coordonné le second sur la genèse d'un Empire (1978), rédigeant en particulier une conclusion très problématisée sur l'impérialisme romain. Il a en outre fondé une école qui a été sans conteste la plus importante en histoire romaine en France d'un point vue quantitatif comme qualitatif et qui y a eu le plus d'impact sur la perception actuelle de la République romaine. C'est ce qui explique une initiative récente consistant à dresser un bilan historiographique à propos du métier de citoyen une quarantaine d'années après sa parution et qui paraîtra en 2020 sous la forme d'un dossier dans les Cahiers du Centre Glotz coordonné par Pascal Montlahuc. Nicolet s'inscrivait dans une démarche de comparatisme historique et d'ouverture aux sciences sociales ${ }^{19}$, en commençant par l'étude de l'organisation civique et de la nature de l'État antique, au travers notamment des recherches sur la fiscalité. On peut ainsi distinguer quatre grands moments dans la conduite de ses recherches.

10 Le premier correspondait à sa thèse sur l'ordre équestre, qui venait prendre place dans le débat, alors vif ${ }^{20}$, qui portait sur la définition des regroupements sociaux, d'ordres ou de classes dans les sociétés modernes ${ }^{21}$. Il eut recours à la méthode prosopographique qui lui permettait de définir le groupe qu'il étudiait. Le résultat était une redéfinition 
d'une catégorie importante de la société romaine dans une classification civique de l'honneur et de la responsabilité.

11 L'œuvre de Nicolet se prolongea dans la rédaction du Métier de citoyen ${ }^{22}$, où il détaillait les mécanismes de l'appartenance civique et du fonctionnement des organes politiques en en soumettant l'analyse à la logique de la répartition des charges et des pouvoirs selon les principes de l'égalité géométrique. L'analyse révélait comment les acteurs, dans l'interaction, mettaient en œuvre des principes et des références collectives. La démarche s'inscrivait ainsi dans une anthropologie politique, mais qui prenait au sérieux - et au mot - les auteurs anciens, et particulièrement Polybe ${ }^{23}$. Pour la première fois était mis en lumière le quotidien des institutions, ainsi que leur vécu dans le fonctionnement du corps civique romain, ce qui préfigurait les travaux actuels sur la communication politique.

Parallèlement, Claude Nicolet poursuivit ses études sur la fiscalité en lien avec la naissance d'un État, dont les plus importants ont été rassemblés dans l'ouvrage intitulé Rendre à César. Économie et société dans la Rome antique (1988) ${ }^{24}$. Dans un autre livre important, Les structures de l'Italie romaine $e^{25}$, il analysait les données géographiques, économiques, fiscales et militaires de la domination romaine et consacrait quelques chapitres à un tableau des institutions.

13 Sa préoccupation principale était en fait l'étude de l'État romain. D'où les deux grands ensembles d'analyses qui suivirent. Il faut tout d'abord mentionner l'Inventaire $d u$ monde ${ }^{26}$. Ce livre remarquable était consacré à la perception par les gouvernants romains de l'espace géographique qu'ils avaient à dominer et à administrer, ainsi qu'aux moyens qu'ils se donnaient pour y parvenir. Nicolet participa par ailleurs aux réflexions sur la naissance de l'État moderne et sur les mégapoles méditerranéennes qui lui permettaient d'engager l'étude des questions d'administration, de gestion et d'approvisionnement de la ville de Rome $^{27}$. Il faut souligner à ce propos à quel point la prise en compte de la dimension spatiale de l'action politique est profondément inscrite dans la tradition historiographique française. Les cursus des universités françaises associent le plus souvent l'Histoire et la Géographie car, les deux disciplines sont enseignées dans les lycées par les mêmes enseignants ayant reçu une double formation. Ce lien fort entre deux disciplines pourtant bien distinctes, si spécifique quand on compare l'enseignement de l'histoire en France et dans les autres pays européens, est à l'origine d'une sensibilité à la topographie de Rome, les monographies sur tel quartier ou telle colline de Rome étant une spécificité de l'historiographie française depuis le livre de M. Besnier sur l'île Tibérine (1902) jusqu'à la thèse de J. Prim sur l'Aventin ${ }^{28}$. Une partie de la réflexion de $\mathrm{M}$. Bonnefond-Coudry porte sur les lieux de réunion du Sénat et sur les règles institutionnelles en vigueur en la matière; particulièrement représentatif de cette tendance est un de ses articles, repris dans le choix opéré pour Trivium, qui met en évidence la dimension politique du parcours suivi au moment du départ et du retour du magistrat pour sa province - ainsi que sur la signification politique des modifications de ce parcours à l'époque d'Auguste ${ }^{29}$.

\section{L'École fondée par Nicolet}

L'apport de Claude Nicolet fut décisif parce qu'il a ouvert la voie à d'autres chercheurs en formant un groupe numériquement important de savants. Nul ne peut prétendre aujourd'hui être à lui seul l'héritier de Nicolet. Il y eut en effet non pas un seul, mais 
une multitude d'héritiers, dont certains ont franchi les barrières chronologiques de la période républicaine pour prolonger les thématiques et les méthodes de leur maître. Le meilleur exemple est celui de Ségolène Demougin, qui a consacré sa thèse d'État également à l'ordre équestre en continuant à fonder son analyse sur une enquête prosopographique préalable ${ }^{30}$, mais en traitant de la période julio-claudienne ${ }^{31}$; dans le même ordre d'idées, les travaux de Jérôme France sur la fiscalité32 et ceux de Pierre Cosme sur l'armée portent essentiellement sur l'époque impériale ${ }^{33}$. Pour la période républicaine, il faut distinguer plusieurs champs d'études qui furent l'occasion de recherches neuves et approfondies dans le prolongement des axes que son œuvre avait définis.

Le premier que nous retenons est celui des études sur l'Italie romaine. Elles étaient et restèrent très riches. Il suffit de penser aux chercheurs italiens, mais aussi aux juristes français et tout particulièrement à Michel Humbert et à Yan Thomas ${ }^{34}$, dont il a déjà été question supra. On pourrait y ajouter les ouvrages de David Kremer sur le droit latin ${ }^{35}$, de Jean-Claude Lacam sur les pratiques religieuses chez les peuples de la péninsule ${ }^{36}$ et de Stéphane Bourdin sur les peuples de l'Italie préromaine (VIII ${ }^{\mathrm{e}} \mathrm{I}^{\mathrm{er}} \mathrm{s}$. av. J.-C. $)^{37}$. De ce fait, notre connaissance de l'Italie pré-romaine et de la Rome des $\mathrm{V}^{\mathrm{e}}$ et $\mathrm{IV}^{\mathrm{e}}$ siècles en est ressortie passablement améliorée et affinée. Les travaux de Mireille Cebeillac-Gervasoni sur les élites municipales s'inscrivirent dans la continuité de sa thèse sur les magistrats des cités du Latium et de Campanie ${ }^{38}$. Elle appliqua à ces personnages la méthode prosopographique. Elle poursuivit ensuite l'étude de cette catégorie sociale par une série de colloques qui lui permirent de proposer un tableau très informé ${ }^{39}$.

Le second champ de recherches que les travaux de Claude Nicolet ont ouvert se situait dans la lignée de ses études sur les appartenances et le fonctionnement des instances civiques. Il faut citer pour commencer les recherches de Philippe Moreau ${ }^{40}$ qui s'est consacré à l'étude des définitions familiales, mais aussi à des analyses très précises des procédures législatives. Elles rejoignent ainsi une partie des travaux de Jean-Louis Ferrary ${ }^{41}$ sur les institutions et les théories politiques romaines et ceux de Marianne Bonnefond-Coudry ${ }^{42}$, dont la thèse sur le Sénat avait pour point de départ l'analyse des procédures de prise de décision. Le poids de l'histoire des institutions est resté important en France durant ces dernières décennies. Une des réalisations en cours est le projet LEges POpuli Romani (LEPOR), porté par J.-L. Ferrary et Ph. Moreau, qui est une base de données reprenant et étendant de manière substantielle le travail de G. Rotondi de 1912 sur les lois des Romains et qui prévoit d'arriver à environ 880 notices, dont chacune sera consacrée à une loi du peuple romain ${ }^{43}$. Il faut ici redire à propos de l'influence exercée par Nicolet à quel point celui-ci resta fondamentalement un historien des institutions, même s'il savait parfaitement que les institutions de la République romaine n'obéissaient pas à des règles aussi strictes qu'on avait bien voulu le faire croire et étaient loin d'avoir été figées à un moment ou un autre. Il a en outre montré que la vie politique reposait aussi sur ce qu'il appelait « les langages parallèles » $(\text { contio, jeux ... })^{44}$ et ouvert ainsi la voie aux travaux sur le mode de communication politique entre l'aristocratie et l'ensemble du corps civique, étant ainsi lui-même à l'origine d'une évolution historiographique que l'on a qualifié de "communicative turn » et qui a été particulièrement fécond en Allemagne. Jérôme France et Frédéric Hurlet viennent de publier en ce sens un manuel sur les institutions romaines dans lequel ils recommandent de ne pas nier l'existence de règles partagées par l'ensemble 
des membres de la res publica tout en précisant qu'il faut aller au-delà d'une histoire des institutions pour comprendre dans sa globalité le fonctionnement du politique ${ }^{45}$.

La méthode de prosopographie sociale pratiquée par Nicolet fut élargie à d'autres catégories. Nous avons déjà cité les travaux de Mireille Cebeillac-Gervasoni sur les «élites » municipales. Il faut ajouter ceux de deux autres élèves de Nicolet: François Hinard, qui a analysé dans sa thèse d'État une procédure apparue lors des guerres civiles, les proscriptions, à partir d'un catalogue prosopographique ${ }^{46}$; Elizabeth Deniaux, qui a repris la question des liens entre clientélisme et pouvoir essentiellement à partir de la correspondance de Cicéron ${ }^{47}$. Dans ce dernier cas, la prosopographie des correspondants de l'orateur permettait d'établir les positions sociales des individus concernés et d'en analyser l'inégalité relative dans les interactions que révélaient ces lettres. Cela permettait des analyses fines du rapport entre rang social et pouvoir, ainsi que des formes stylistiques de son expression. L'application de la méthode prosopographique aux orateurs qui avaient plaidé dans des procès a permis à JeanMichel David d'abord de faire apparaitre les liens entre ce type d'intervention civique et la position qu'ils occupaient dans la cité, mais elle poussait aussi à identifier les relations entre statut et conduite oratoire ${ }^{48}$, de telle sorte que pouvaient apparaître les diverses facettes d'un ethos aristocratique. C'est ainsi que se mit en place une convergence avec les analyses qui avaient été développées en Allemagne, dans la lignée de Christian Meier, autour des notions de grammaire ou de culture politique ${ }^{49}$. C'est dans cette perspective que Jean-Michel David a écrit l'article sur les manifestations de violence collective qui est incorporé dans ce numéro de Trivium $^{50}$.

18 La prosopographie sociale continue à être pratiquée par les historiens français de la République romaine, mais avec la conscience qu'elle n'est qu'une étape, et un moyen d'obtenir des résultats dépassant la mise en série de personnages ayant un point en commun. Cette méthode a été suivie par plusieurs des élèves de Jean-Michel David et de Jean-Michel Roddaz, dont les thèses reposent sur des enquêtes prosopographiques relatives à différents acteurs de la vie civique : les patriciens (Robinson Baudry) ${ }^{51}$; les tribuns de la plèbe (Thibaud Lanfranchi) ${ }^{52}$; les infâmes (Clément Bur) ${ }^{53}$; les Scipions (Henri Etcheto, qui analysa les stratégies de construction et de reproduction du pouvoir et de la légitimité d'une famille romaine) ${ }^{54}$; les partisans de Marc Antoine (Marie-Claire Ferriès, qui traite des conditions du recrutement et le fonctionnement de l'adhésion à un chef) ${ }^{55}$. Elle est en outre également au centre d'un projet actuellement en cours, «le Broughton ${ }^{56}$ augustéen ", piloté par Robinson Baudry et Frédéric Hurlet, qui veut étendre les fastes à l'ensemble de l'époque augustéenne et qui reprendra également toutes les données sur les magistrats d'époque triumvirale.

La dernière grande direction de recherches que Nicolet avait ouverte sur l'administration de l'Empire et particulièrement celle de la ville de Rome fut empruntée par plusieurs auteurs: Pierre Cosme sur la gestion de l'armée, mais principalement pour l'époque impériale comme il a déjà été $\mathrm{dit}^{57}$; Catherine Virlouvet, qui établit une passerelle entre histoire économique et sociale en étudiant les procédures de distribution gratuite de blé au peuple à Rome à la fin de la République et au début de l'époque impériale, ainsi que le ravitaillement de la ville de Rome ${ }^{58}$. Ces études avaient pour effet de poser la question d'une modernité de l'État romain ${ }^{59}$. On mentionnera pour finir les réalisations d'un programme de recherche que Nicolet avait lancé et qui portait sur les archives en tant que production écrite découlant du fonctionnement d'un État. Claudia Moatti a prolongé de tels travaux d'abord en étudiant un tel aspect 
dans sa thèse de doctorat consacrée au partage de la terre dans le monde romain (II ${ }^{\mathrm{e}}$ siècle av. J.-C. - $\mathrm{I}^{\mathrm{er}}$ siècle ap.J.-C.), puis en prenant en charge avec Ségolène Demougin le programme de recherche intitulée " La mémoire perdue ${ }^{60}$ ».

À côté de ces travaux poursuivant les recherches de Nicolet, il faut signaler d'autres prolongements qui portaient sur la fin de la République et la mise en place du Principat. Jean-Michel Roddaz étudia en particulier à travers le personnage d'Agrippa comment la période triumvirale et le principat augustéen s'inscrivent dans la continuité des institutions de la res publica, mais aussi à quel point ils constituent un temps de transition, et par bien des aspects de rupture dans l'exercice du pouvoir ${ }^{61}$. Frédéric Hurlet a commencé par mener une réflexion sur la dictature de Sylla - sa légitimité et sa légalité - pour étudier ensuite la mise en place de la monarchie augustéenne en s'intéressant à ceux qu'on appelle les «co-régents " (Agrippa, Drusus l'Ancien, Caius et Lucius César, Tibère, Germanicus et Drusus le Jeune) et en s'appuyant sur l'étude des comportements politiques pour rendre compte de la constitution des normes $^{62}$. Il a défendu de manière générale l'idée que le principat augustéen constituait l'ultime chapitre de l'histoire de la République romaine et devait être étudié comme une période de transition qui serait incompréhensible si l'on ne mesurait pas le poids de la République - de ses formes, mais aussi de son prestige et de sa mémoire - dans la création d'un nouveau régime. Il a montré en particulier dans quelle mesure la res publica d'Auguste s'inscrit dans le prolongement formel des institutions, des valeurs et des pratiques de la République romaine tout en devenant un régime politique autre, de nature foncièrement monarchique. Un des meilleurs exemples est l'étude qui a été reprise par ce numéro de Trivium dans la liste des articles traduits et dans laquelle il montre comment le phénomène de la concurrence si caractéristique de la République romaine perdura sous Auguste en s'adaptant à la nouvelle forme monarchique du pouvoir : la compétition était désormais arbitrée non plus par le peuple, mais par le prince $^{63}$.

\section{Au-delà de la crise de la République romaine. La République archaïque et moyenne}

À la fin des années quatre-vingt-dix du siècle passé et au cours des deux décennies suivantes, d'autres auteurs apparurent, qui s'inscrivaient pour l'essentiel dans la continuité des précédents, mais avec des évolutions notables en matière de périodisation. Nicolet considérait que l'état de la documentation ne permettait pas d'écrire une véritable histoire de la République romaine avant le début du $\mathrm{III}^{\mathrm{e}}$ siècle. Le jugement était excessif, comme le montrent l'existence de livres importants publiés sur l'époque médio-républicaine et la vitalité des débats actuels sur le $\mathrm{V}^{\mathrm{e}}$ siècle, mais il a tout de même contribué à orienter ses élèves principalement vers l'étude de l'époque tardo-républicaine. Cette situation explique que l'on continue à s'intéresser à la fin de la République et à la notion même de crise, mais elle a tout de même passablement évolué et on notera le renouveau des études sur la République archaïque et moyenne. Dominique Briquel, sans doute l'un des rares antiquisants à continuer à utiliser les travaux de Georges Dumézil, a consacré aux débuts de la République romaine des analyses érudites aussi bien que des études de synthèse ${ }^{64}$. Les travaux de Michel Humm n'ont sans doute pas réglé de façon définitive la question complexe de la transition de la royauté à la république, mais ils ont avancé à ce sujet de nouvelles hypothèses 
importantes en étudiant le cas de Rome à l'aune des évolutions qui avaient lieu au même moment dans les autres cités d'Italie ${ }^{65}$. Ils ont également contribué à mieux connaître la figure d'Appius Claudius Caecus ${ }^{66}$ et à montrer comment l'organisation des magistratures romaines - le cursus honorum - se mit progressivement en place $\mathrm{du}^{\mathrm{e}} \mathrm{au}$ III siècle av. J.-C. ${ }^{67}$. Ils ont été complétés par plusieurs thèses de doctorat qui ont étudié le processus de formation de la res publica romaine et l'histoire de l'Italie avant le $\mathrm{III}^{\mathrm{e}}$ siècle. Si la thèse de Robinson Baudry sur les patriciens porte sur une période plus récente, elle a naturellement été amenée à étudier à nouveaux frais la lutte politique qui opposa au début de la République les patriciens aux plébéiens aux V ${ }^{\mathrm{e}}$ et $\mathrm{IV}^{\mathrm{e}}$ siècles $^{68}$; quant à Thibaud Lanfranchi, il a choisi comme borne chronologique de sa thèse sur les tribuns de la plèbe les années 494 et 287 et souligné leur rôle dans la construction des instances où le peuple pouvait exercer un pouvoir ${ }^{69}$.

\section{Au-delà de l'histoire des institutions. La culture politique de la République romaine}

Pour les travaux les plus récents, il faut souligner l'intérêt d'études sur des pratiques civiques qui rejoignent les études menées en Allemagne sur la notion de culture politique et ou s'en ont inspirées. On signalera à ce sujet deux thèses, publiées : celle sur le triomphe de Jean-Luc Bastien tout d'abord, qui analyse les rituels de cette cérémonie, son insertion et son rôle dans le contexte politique ${ }^{70}$; celle aussi de Yann Berthelet sur les prises d'auspices et les modalités de la prise en compte de la volonté des dieux et des pratiques religieuses dans le fonctionnement de la société tout entière et les choix politiques faits par la cité ${ }^{11}$.

L'étude de différentes catégories sociales constitutives et actrices de la cité se poursuit avec un intérêt particulier pour l'analyse des comportements et les représentations. Il faut citer ici les travaux de Jean-Pierre Guilhembet ${ }^{72}$ sur la domus aristocratique et l'espace urbain dont nous avons repris un article dans cette publication ${ }^{73}$ et dans un domaine voisin le livre de Cyril Courrier ${ }^{74}$ sur la population de la Ville, ses conditions de vie, ses représentations (sa culture) et son rôle politique. François $\mathrm{Cadiou}^{75}$ a repris la question de la place de l'armée dans le fonctionnement de la communauté, de son recrutement et de son rôle politique, en démontant tous les présupposés historiographiques qui sont à l'origine de l'idée de «prolétarisation » de l'armée et en insistant sur la continuité de la définition civique qui était la sienne. Clément Bur a étudié les mécanismes de définition de l'infamie et de la dégradation symbolique ${ }^{76}$. Enfin, dans la lignée des études sur les entourages mais en l'associant à la prise en compte de la poésie comme une pratique sociale et culturelle, Philippe Le Doze a soumis le groupe de Mécène à une analyse qui éclaire les modes de célébration du princeps et l'influence politique qui en découle ${ }^{77}$. Il faut noter enfin l'intérêt des études des comportements à partir de celle des valeurs qui leur servaient de référence. Quatre livres récents répondent à cette définition : celui de Philippe Akar sur la Concordia ${ }^{78}$; celui de Mathieu Jacotot sur l'honneur ${ }^{79}$; celui de Claudia Moatti sur la notion de res publica $^{80}$; enfin l'ouvrage collectif qui fait de l'auctoritas une notion essentielle dans la prise de décision et qui a été coordonné par Jean-Michel David et Frédéric Hurlet ${ }^{81}$.

Ces ouvrages sont en grande partie ceux d'une nouvelle génération qui s'inscrit dans la continuité de la précédente, mais qui marque aussi sa différence. Comme leurs immédiats prédécesseurs, ces auteurs conservent dans leurs analyses la distance 
critique qui permet de traiter la société romaine de la République dans son altérité. Mais leur démarche d'ensemble est beaucoup moins soumise aux enjeux théoriques qu'elle ne l'était dans les années 60 à 80 du siècle passé. Il est frappant d'ailleurs de constater que bien peu d'historiens français de la République romaine sont entrés dans le débat épistémologique qui a opposé Fergus Millar aux collègues allemands, au premier rang desquels, Karl-Joachim Hölkeskamp ${ }^{82}$, sur les modes d'exercice de la politique dans la République romaine. Outre les comptes rendus de Stéphane Benoist ${ }^{83}$ et de Claudia Moatti ${ }^{84}$, on ne peut guère relever que les contributions de Frédéric Hurlet $^{85}$ et Jean-Michel David ${ }^{86}$. Pourtant, malgré cette absence de référence explicite, la réflexion de l'historiographie française reste inspirée par les sciences sociales et plus particulièrement par la sociologie ${ }^{87}$. Deux champs semblent désormais l'emporter, celui des comportements et celui des systèmes de référence par rapport auxquels ils se déterminaient, ce qui nous renvoie à cette notion de culture politique qui, pour les recherches qui ambitionnent de rendre compte du fonctionnement de la société et non pas simplement d'établir des faits, devient un cadre important, revendiqué ou non, de la démarche historique. Mais en même temps, ces travaux évitent le plus souvent le formalisme sévère d'une analyse qui se limiterait à l'étude des conduites. Les groupes d'acteurs (aristocratie, plèbe, armée, etc.) sont définis dans leur composition concrète et ne s'effacent pas derrière les pratiques qui les caractérisent ou les rituels civiques auxquels ils participent. Les résultats de ces travaux ont permis d'aller définitivement au-delà de l'histoire des institutions.

Le principal enjeu est désormais de mieux comprendre les pratiques politiques de manière à reconstituer ce que l'on peut appeler une grammaire du politique dans la Rome de la République. Une telle notion s'est développée à partir des travaux de JeanMichel David, qui a montré à quel point le pouvoir aristocratique reposait aussi sur une axiologie des comportements et sur des notions extra-institutionnelles. Il en a résulté une ouverture des historiens actuels de la République romaine vers les sciences sociales, dont l'apport est désormais davantage pris en compte. On relit aujourd'hui plus qu'avant les œuvres des sociologues tels que M. Weber, A. Kojève, J. Habermas ou encore P. Bourdieu de manière non pas à appliquer servilement à l'Antiquité leurs modèles théoriques, mais à les adapter et à les ajuster. Cette nouvelle manière de concevoir l'histoire de la République romaine conduit à la publication d'ouvrages, individuels et collectifs, sur des notions telles que le prestige (Robinson Baudry et Frédéric Hurlet) ${ }^{88}$, la norme (Tanja Itgenshorst et Philippe Le Doze) ${ }^{89}$, le charisme (Raphaëlle Laignoux et Pascal Montlahuc) ${ }^{90}$, l'opinion publique (Frédéric Hurlet et Pascal Montlahuc) ${ }^{91}$, de nouveau la crise (B. Augier et R. Baudry) ${ }^{92}$, ou sur des pratiques comme le rire (et le faire rire) dans la vie politique (Pascal Montlahuc) ${ }^{93}$. Cela signifie que l'histoire sociale reste, conformément à une tradition bien établie de l'historiographie française, un fondement essentiel de l'analyse. Et cette continuité permet de penser que les recherches à venir conserveront cette ambition essentielle de l'Historien, de rendre compte de tous les aspects et de tous les mécanismes d'une société. 


\section{En guise d'épilogue : les historiographies française et allemande en regard}

Il faut ajouter pour finir quelques réflexions sur les relations de l'historiographie française avec l'historiographie allemande sur la République romaine. La question est assurément complexe, la question de la langue constituant dans ce cas une barrière. Il faut préciser d'emblée qu'il est impossible de généraliser et qu'il faut compter avec la sensibilité de chacun dans le développement ou non des relations entre ces deux historiographies. C'est ainsi que $\mathrm{Cl}$. Nicolet n'a jamais travaillé ni de près ni de loin avec Chr. Meier, qui n'a été invité à Paris par des historiens que par l'entremise de P. Veyne ${ }^{94}$. En revanche, J.-M. David est à l'origine du colloque de Strasbourg de 1997 portant sur la fin de la République romaine et conçu comme "un débat francoallemand d'histoire et d'historiographie »; il s'est associé à un collègue allemand travaillant au CNRS et parfaitement inséré dans les deux communautés scientifiques, $\mathrm{H}$. Bruhns $\mathrm{s}^{95}$. Plus récemment, une collègue allemande nommée comme professeur à l'Université de Reims, T. Itgenshorst, a organisé avec un collègue français, Ph. Le Doze, un colloque consacré à la question de la norme sous la République romaine et le HautEmpire et rassemblant les meilleurs spécialistes français, belges et allemands. Il faut noter également que l'essai de K.-J. Hölkeskamp sur la République romaine paru en 2004 a été traduit en français en 2008, réalisation qui mérite d'être soulignée dans un pays où l'on traduit plutôt rarement des ouvrages scientifiques publiés dans d'autres langues. L'initiative de la revue Trivium est donc un signe de la vitalité des échanges qui existent entre ces deux historiographies et dont on peut raisonnablement penser qu'ils vont perdurer pour les années et les décennies à venir.

\section{BIBLIOGRAPHIE}

Akar, Ph. (2013) : Concordia, un idéal de la classe dirigeante romaine à la fin de la République, Paris.

Andreau, J. (1974) : Les affaires de Monsieur Jucundus, Rome.

Andreau, J. (1987) : La vie financière dans le monde romain, les métiers de manieurs d'argent : IVe siècle av. J.-C. - III siècle ap. J.-C., Rome.

Andreau, J. (1997) : Patrimoines, échanges et prêts d'argent, l'économie romaine, Rome.

Andreau, J. (2010) : L'économie du monde romain, Paris.

Assenmaker, P. (2014) : De la victoire au pouvoir. Développement et manifestations de l'idéologie impératoriale à l'époque de Marius et Sylla, Bruxelles.

Augier, B. / Baudry, R. / Rohr Vio, Fr. (éd.) (à paraître) : La crise, quelle(s) crises ? Nouvelles lectures politiques de la République tardive, des Gracques à la mort de César, Rome.

Barrandon, N. (2011) : De la pacification à l'intégration des Hispaniques (133-27 a.C.). Les mutations des sociétés indigènes d'Hispanie centrale et septentrionale sous domination romaine, Bordeaux. 
Barrandon, N. (2018) : Les massacres de la République romaine, Paris.

Barrandon, N. / Kirbihler, Fr. (éd.) (2010) : Administrer les provinces de la République romaine, Rennes.

Barrandon, N. / Kirbihler, Fr. (éd.) (2011) : Les gouverneurs et les provinciaux sous la République romaine, Rennes.

Bastien, J.-L. (2007) : Le triomphe romain et son utilisation politique à Rome aux trois derniers siècles de la République, Rome.

Baudry, R. (2008) : Les patriciens à la fin de la République romaine et au début du Principat, thèse Paris 1.

Baudry, R. (2017) : « Les familles de l'aristocratie romaine », Pallas, p. 209-226.

Baudry, R. / Hurlet, Fr. (éd.) (2016) : Le prestige à Rome à la fin de la République et au début du Principat, Paris.

Bayet, J. (1957) : Histoire politique et psychologique de la religion romaine, Paris.

Benoist, St. (2010) : « c.r. Hölkeskamp K.-J., Reconstruire une République, la « culture politique » de la Rome antique et la recherche des dernières décennies ", RH, 653, p. 162-164.

Berrendonner, Cl. / Cébeillac-Gervasoni, M. / Lamoine, L. (éd). (2008) : Le quotidien municipal dans l'Occident romain, Clermont-Ferrand.

Berrendonner, Cl. / Cébeillac-Gervasoni, M. / Lamoine, L. (éd). (2010) : La Praxis dans l'Occident romain, Clermont-Ferrand.

Berthelet, Y. (2015) : Gouverner avec les dieux, autorité, auspices et pouvoir sous la République romaine et sous Auguste, Paris.

Bonnefond-Coudry, M. (1987) : « Transferts de fonctions et mutation idéologique : le Capitole et le Forum d'Auguste ", in : L'Vrbs. Espace urbain et histoire (Ir siècle av. J.-C. - III siècle ap. J.-C.), Rome, p. 251-278.

Bonnefond-Coudry, M. (1989) : Le Sénat de la République romaine de la guerre d'Hannibal à Auguste, Rome.

Bourdin, St. (2012) : Les peuples de l'Italie préromaine : identités, territoires et relations inter-ethniques en Italie centrale et septentrionale (VIII ${ }^{e} \mathrm{I}^{\text {er }}$ s. av. J.-C.), Rome.

Briquel, D. (2000) : dans Histoire romaine, t. I (Des origines à Auguste), Paris, p. 11-243.

Bruhns, H. / David, J. M. / Nippel, W. (éd.) (1997): Die späte römische Republik - La fin de la république romaine. Un débat franco-allemand d'histoire et d'historiographie, Rome.

Bur, Cl. (2018) : La citoyenneté dégradée, une histoire de l'infamie à Rome (312 av. J.-C. - 96 apr. J.-C.), Rome.

Cadiou, Fr. (2008) : Hibera in terra miles. Les armées romaines et la conquête de l'Hispanie sous la république (218-45 av.J.-C.), Madrid.

Cadiou, Fr. (2018) : L'armée imaginaire, les soldats prolétaires dans les légions romaines au dernier siècle de la République, Paris.

Cébeillac-Gervasoni, M. (1998) : Les magistrats des cités italiennes de la seconde guerre punique à Auguste : le Latium et la Campanie, Rome.

Cébeillac-Gervasoni, M. / Lamoine, L. (éd). (2003) : Les élites et leurs facettes : les élites locales dans le monde hellénistique et romain, Rome. 
Cébeillac-Gervasoni, M. / Lamoine, L. / Trément, Fr. (éd). (2004) : Autocélébration des élites locales dans le monde romain : contextes, textes, images (II ${ }^{e}$ s. av. J.-C. - III ${ }^{e}$ s. ap. J.-C.), Clermont-Ferrand. Chouquer, G. / Favory, Fr. (1980) : Contribution à la recherche des cadastres antiques, Paris.

Chouquer, G. / Favory, Fr. (1991) : Les paysages de l'Antiquité, terres et cadastres de l'Occident romain, $I V^{e}$ siècle avant J.-C.-III ${ }^{e}$ siècle après J.-C., Paris.

Clavel-Lévêque, M. (1970) : Béziers et son territoire dans l'Antiquité, Paris.

Cosme, P. (2007) : L'armée romaine, VIII s. av. J.-C.-Vee s. ap. J.-C., Paris.

Courrier, C. (2014) : La plèbe de Rome et sa culture (fin du II siècle av. J.-C. - fin du I ${ }^{e r}$ siècle ap. J.-C.), Rome.

David, J.-M. (1992) : Le patronat judiciaire au dernier siècle de la République romaine, Rome.

David, J.-M. (2006) : « Una repubblica in cantiere », Studi Storici, 47 (2), p. 365-376.

David, J.-M. (2011) : «Claude Nicolet : Le métier de citoyen et les structures de l'Italie romaine », in : David, J.-M. / Demougin, S. / Deniaux, E. / Ferrary, J.-L. / Virlouvet, C. (éd.) : Claude Nicolet, Historien et Citoyen, CCG, 22, p. 95-104.

David, J.-M. (2019a) : Au service de l'honneur. Les appariteurs de magistrats romains, Paris.

David, J.-M. (2019b) : «Préface » à la nouvelle édition du Patronat judiciaire au dernier siècle de la République romaine, Rome, p. IX-XVII.

David, J.-M. / Hurlet, Fr. (éd.) (à paraître) : L'auctoritas à Rome. Un élément constitutif de la culture politique, Bordeaux.

Demougin, S. (1988) : L'ordre équestre sous les Julio-Claudiens, Rome.

Demougin, S. (1992) : Prosopographie des chevaliers romains julio-claudiens (43 av. J.-C. - 70 ap. J.-C.),

Rome.

Demougin, S. (éd.) (1994) : La mémoire perdue. À la recherche des archives oubliées, publiques et privées, de la Rome antique, Rome.

Deniaux, E. (1993) : Clientèles et pouvoir à l'époque de Cicéron, Rome.

Dubuisson, M. (1985) : Le Latin de Polybe. Les implications historiques d'un cas de bilinguisme, Paris.

Etcheto, H. (2012) : Les Scipions, famille et pouvoir à l'époque républicaine, Bordeaux.

Famerie, Ét. (1996) : Le latin et le grec d'Appien. Contribution à l'étude du lexique d'un historien grec de Rome, Genève.

Ferrary, J.-L. (2012) : Recherches sur les lois comitiales et sur le droit public romain, Pavie.

Ferrary, J.-L. (2014 [1988]) : Philhellénisme et impérialisme. Aspects idéologiques de la conquête romaine du monde hellénistique de la seconde guerre de Macédoine à la guerre contre Mithridate, $2^{\mathrm{e}}$ éd., Rome.

Ferrary, J.-L. (2017) : Rome et le monde grec. Choix d'écrits, Paris.

Ferriès, M.-Cl. (2007) : Les partisans d'Antoine (des orphelins de César aux complices de Cléopâtre), Bordeaux.

France, J. (2001) : Quadragesima Galliarum. L'organisation douanière des provinces alpestres, gauloises et germaniques de l'Empire romain (Irr siècle avant J.-C.-III ${ }^{e}$ siècle après J.-C.), Rome.

France, J. / Hurlet, Fr. (2019) : Institutions romaines, Paris. 
Gonzales, A. (2015a) : «Esclavage, universalisme et cosmopolitisme stoïciens entre cité idéale et histoire », XXXV Coloquio internacional del GIREA, Madrid, p. 463-485.

Gonzales, A. (2015b) : « Du praedium au fundus. Proscriptions, expropriations et confiscations chez les Agrimensores romains : problèmes techniques et juridiques », MEFRA, 127 (2) [en ligne].

Guilhembet, J.-P. (2016a) : « Domus et monumenta : la résidence urbaine et ses pouvoirs de mémoire dans la ville de Rome (fin de la République - Haut Empire) », in : Benoist, St. / DaguetGagey, A. / Hoët-van Cauwenberghe, Chr. (éd.) : Une mémoire en actes : espaces, figures, discours dans le monde romain, Villeneuve d'Ascq, p. 77-88.

Guilhembet, J.-P. (2016b) : « La domus instrument de prestige aristocratique », in : Baudry, R. / Hurlet, Fr. (éd.) : Le prestige à Rome à la fin de la République et au début du Principat, Paris, p. 179-191.

Guilhembet, J.-P. / Laignoux, R. / Montlahuc, P. (éd.) (à paraître) : Le charisme dans l'Antiquité, Rome.

Hinard, F. (1985) : Les Proscriptions de la Rome républicaine, Rome.

Hinard, F. / Briquel, D. / Brizzi, G. / Roddaz, J.-M. (éd.) (2000) : Histoire romaine. Tome I (Des origines à Auguste), Paris.

Hölkeskamp, K.-J. (2008) : Reconstruire une République, la « culture politique » de la Rome antique et la recherche des dernières décennies, Nantes (orig. 2004).

Humbert, M. (1993) : Municipium et Civitas sine suffragio, l'organisation de la conquête jusqu'à la guerre sociale, Rome.

Humbert, M. (éd.) (2005) : Le Dodici Tavole, dai Decemviri agli Umanisti, Pavie.

Humbert, M. (2013) : Antiquitatis effigies, recherches sur le droit public et privé de Rome, Pavie.

Humbert, M. (2018) : La loi des XII Tables. Édition et commentaire, Rome.

Humm, M. (2005) : Appius Claudius Caecus, la République accomplie, Rome.

Humm, M. (2012) : « Hiérarchie des pouvoirs et hiérarchie des magistratures dans la Rome républicaine ", in : Bérenger, A. / Lachaud, Fr. (éd.) : Hiérarchie des pouvoirs, délégation de pouvoir et responsabilité, Metz, p. 105-134.

Humm, M. (2017a) : « La Regia, le rex sacrorum et la Res publica », Archimède. Archéologie et histoire ancienne [en ligne], 4, p. 129-154.

Humm, M. (2017b) : « Du “dualisme patricio-pébéien” à la société d'ordres. La République romaine de la fin du VIe à la fin du IV e siècle av. J.-C. », in : Moatti, Cl. / Müller, Chr. (éd.) : Statuts personnels et espaces sociaux. Questions grecques et romaines, Paris, p. 45-84.

Hurlet, Fr. (1993) : La dictature de Sylla, monarchie ou magistrature républicaine? Essai d'histoire constitutionnelle, Bruxelles / Rome.

Hurlet, Fr. (1997) : Les collègues du prince sous Auguste et Tibère. De la légalité républicaine à la légitimité dynastique, Rome.

Hurlet, Fr. (2012a) : « Démocratie à Rome ? Quelle démocratie ? En relisant Millar (et Hölkeskamp) », in : Benoist, St. (éd.) : Rome, a City and its Empire in Perspective: The Impact of the Roman World through Fergus Millar's Research, Leyde, p. 19-43.

Hurlet, Fr. (2012b) : « Concurrence gentilice et arbitrage impérial. Les pratiques politiques de l'aristocratie augustéenne ", Politica Antica. Rivista di prassi e cultura politica nel mondo greco e romano, 2, p. 33-54. 
Hurlet, Fr. / Montlahuc, P. (2018) : «L'opinion publique dans la Rome tardo-républicaine. À propos du livre de Cr. Rosillo-López, Public Opinion and Politics in the Late Roman Republic, Cambridge, Cambridge University Press, 2017 », REA, 120, p. 489-507.

Itgenshorst, T. / Le Doze, Ph. (éd.) (2017) : La norme sous la République et le Haut-Empire romains. Élaboration, diffusion et contournements, Bordeaux.

Jacotot, M. (2013) : Question d'honneur, les notions d'honos, honestum et honestas dans la République romaine antique, Rome.

Kremer, D. (2006) : Ius latinum, le concept de droit latin sous la République et l'Empire, Paris.

Lacam, J.-Cl. (2010) : Variations rituelles, les pratiques religieuses en Italie centrale et méridionale au temps de la deuxième guerre punique, Rome.

Laignoux, R. (2014) : « Pour une réévaluation du charisme et de ses usages en sciences sociales », in : Bernardou, V. / Blanc, F. / Laignoux, R. / Roa Bastos, F. (éd.) : Que faire du charisme ? Retours sur une notion de Max Weber, Rennes, p. 13-25.

Lanfranchi, Th. (2015), Les tribuns de la plèbe et la formation de la République romaine, Rome.

Lange, C. H. / Vervaet, Fr. (éd.) (2014) : The Roman Republican Triumph: Beyond the Spectacle, Rome. Le Glay, M. (1971) : La religion romaine, Paris.

Magdelain, A. (1968) : Recherches sur l'« imperium », la loi curiate et les auspices d'investiture, Paris.

Magdelain, A. (1990) : Ius, imperium, auctoritas. Études de droit romain, Rome.

Moatti, Cl. (1993) : Archives et partage de la terre dans le monde romain (II ${ }^{e}$ siècle avant - I ${ }^{e r}$ siècle après J.-C.), Rome.

Moatti, Cl. (1997) : La raison de Rome, naissance de l'esprit critique à la fin de la République (II ${ }^{e}{ }^{e r}$ siècle avant Jésus-Christ), Paris.

Moatti, Cl. (éd.) (1998) : La mémoire perdue. Recherches sur l'administration romaine, Rome.

Moatti, Cl. (2009) : « c.r. Hölkeskamp K.-J., Reconstruire une République, la « culture politique » de la Rome antique et la recherche des dernières décennies ", Annales (HSS), 64 (5), p. 1185-1186.

Moatti, Cl. (2018) : Res publica, Histoire romaine de la chose publique, Paris.

Montlahuc, P. (2019) : Le pouvoir des bons mots : "faire rire" et politique à Rome du milieu du III siècle a.C. jusqu'à l'avènement des Antonins, Rome.

Moreau, Ph. (1982) : Clodiana religio, un procès politique en 61 av. J.-C., Paris.

Moreau, Ph. (2002) : Incestus et prohibitae nuptiae, conception romaine de l'inceste et histoire des prohibitions matrimoniales pour cause de parenté dans la Rome antique, Paris.

Nicolet, Cl. (1966-1974) : L'ordre équestre à l'époque républicaine (312-43 av. J.-C.), Paris.

Nicolet, Cl. (1970) : «Introduction », dans Recherches sur les structures sociales dans l'Antiquité classique, Caen, 25-26 avril 1969, Paris, p. 1-18.

Nicolet, Cl. (1976a) : Le métier de citoyen dans la Rome républicaine, Paris.

Nicolet, Cl. (1976b) : Tributum, Recherches sur la fiscalité directe sous la République romaine, Bonn.

Nicolet, Cl. (1977) : Rome et la conquête du monde méditerranéen. I. Les structures de l'Italie romaine, Paris.

Nicolet, Cl. (1978) : Rome et la conquête du monde méditerranéen. II. Genèse d'un Empire, Paris. 
Nicolet, Cl. (1989) : Rendre à César : Économie et société dans la Rome antique, Paris.

Nicolet, Cl. (1990) : « L’Empire romain est-il un État moderne ? », in : Coulet, N. / Genet, J.-Ph. (éd.) : L'État moderne, le droit, l'espace et les formes de l'État : actes du colloque tenu à La Baume-lès-Aix, 11-12 octobre 1984, Paris.

Nicolet, Cl. (2007) : « Conclusions », in : Genet, J.-Ph. (éd.) : Rome et l'État moderne européen, actes du colloque de Rome, EFR, 31 janvier - 2 février 2002, Rome, p. 419-426.

Nicolet, Cl. / Ilbert, R. / Depaule, J.-Ch. (éd.) (2000) : Mégapoles méditerranéennes à travers les âges. Géographie urbaine rétrospective. Actes du colloque organisé par l'EFR et la MMSH (Rome, 8-11 mai 1996), Rome.

Prim, J. (2018) : « De l'Aventin aux sept collines de Rome : la mémoire des collines comme enjeu de pouvoir entre la fin de la République et le début du Principat ", in : De Souza, M. (éd.) : Les collines dans la représentation et l'organisation du pouvoir à Rome, Bordeaux, p. 83-98.

Roddaz, J.-M. (1984) : Marcus Agrippa, Rome.

Roddaz, J.-M. (1986) : « Guerres civiles et romanisation dans la vallée de l'Ebre », REA, 88, p. 317-338.

Roddaz, J.-M. (1998) : « Les Scipions et l'Hispanie », REA, 100, p. 341-358.

Roman, Y. (1983) : De Narbonne à Bordeaux. Un axe économique au Ir siècle av. J.-C. (125 av. J.-C.-14 apr. J.-C.), Lyon.

Roman, Y. (2016) : Rome, de Romulus à Constantin, histoire d'une première mondialisation, VIII siècle av. J.-C. - IV siècle apr. J.-C., Paris.

Roman, Y. / Roman, D. (1997) : Histoire de la Gaule : VIe siècle av. J.-C. - Ir siècle apr. J.-C. : une confrontation culturelle, Paris.

Scheid, J. (2001 [1985]) : Religion et piété à Rome, Paris.

Scheid, J. (2005) : Quand faire c'est croire, Paris.

Thomas, Y. (1984) : « Mommsen et l'“Isolierung" du droit », Introduction à la réimpression du Droit public, trad. fr., Paris, p. 1-52.

Thomas, Y. (1996) : « Origine » et « commune patrie », étude de droit public romain (89 av. J.-C. - 212 ap. J.-C.), Rome.

Van Haeperen, Fr. (2002) : Le collège pontifical ( $3^{e}$ s. a.C. $-4^{e}$ s. p.C.). Contribution à l'étude de la religion publique romaine, Bruxelles / Rome.

Veyne, P. (1971) : Comment on écrit l'histoire, essai d'épistémologie, Paris.

Veyne, P. (1976) : Le Pain et le cirque : sociologie historique d'un pluralisme, Paris.

Virlouvet, C. (1985) : Famines et émeutes à Rome, des origines de la République à la mort de Néron, Rome.

Virlouvet, C. (1995) : Tessera frumentaria, les procédures de distribution du blé public à Rome à la fin de la République et au début de l'Empire, Rome.

Virlouvet, C. (2009) : La plèbe frumentaire dans les témoignages épigraphique, essai d'histoire sociale et administrative du peuple de Rome antique, Rome. 


\section{NOTES}

1. Sur Claude Nicolet, cf. le numéro de l'année 2011 des Cahiers du Centre Glotz, qui lui a été entièrement consacré.

2. Voir en particulier, Andreau (1974), (1987), (1997), (2010).

3. Voir notamment, Clavel-Lévêque (1970) ; Chouquer / Favory (1980), (1991) ; Gonzales (2015a) et (2015b).

4. En particulier Scheid (2001 [1985]), (2005).

5. Voir en part. Bayet (1957); Le Glay (1971).

6. Cf., exempli gratia, Magdelain (1968) et (1990).

7. Voir en part. Humbert (2005) ; (2013), p. 233-275 et 541-701 ; (2018).

8. Thomas (1984).

9. Cf. notamment Roddaz (1986) et (1998).

10. Barrandon (2011) et (2018); Barrandon-Kirbihler (2010) et (2011).

11. Cadiou (2008).

12. Voir surtout Veyne (1971) et (1976).

13. On citera les noms de Verboven et de Vervaet.

14. Dubuisson (1985).

15. Famerie (1996).

16. Van Haeperen (2002).

17. Assenmaker (2014).

18. Nicolet (1966-1974).

19. Voir David (2011), en part. p. 98-99.

20. Voir Nicolet (1970).

21. Sur le débat alors vif qui portait sur la définition des regroupements sociaux, d'ordres ou de classes, dans les sociétés modernes, voir Nicolet (1970).

22. Nicolet (1976a).

23. David (2011), en part. p. 98-99.

24. Nicolet (1976b).

25. Nicolet (1977)

26. Nicolet (1988).

27. Voir, en particulier, Nicolet (1988), (1990), (2007) ; Nicolet / Ilbert / Depaule (2000).

28. Cf. notamment Prim (2018).

29. Bonnefond-Coudry (1987).

30. Demougin (1988) et (1992).

31. De ce point de vue, elle s'inscrit autant dans la filiation de Nicolet que dans celle de H.-G. Pflaum.

32. Cf. notamment France (2001).

33. Voir en particulier Cosme (2007).

34. Thomas (1996).

35. Kremer (2006).

36. Lacam (2010).

37. Bourdin (2012).

38. Cebeillac-Gervasoni (1998).

39. Cf. notamment Cébeillac-Gervasoni / Lamoine (2003); Cébeillac-Gervasoni Lamoine / Trément (2004) ; Berrendonner / Cébeillac-Gervasoni / Lamoine (2008) et (2010).

40. Voir en particulier Moreau (1982) et surtout (2002).

41. Voir en particulier Ferrary (2012) ; cf. aussi à propos des relations entre Rome et les Grecs Ferrary (2014 [1988]) et (2017).

42. Bonnefond-Coudry (1989). 
43. http://www.cn-telma.fr/lepor/introduction/

44. Nicolet (1976a), p. 456-505.

45. France / Hurlet (2019).

46. Hinard (1985).

47. Deniaux (1993).

48. David (1992) ; cf. aussi David (2019b).

49. Voir Hölkeskamp (2008).

50. David (2013).

51. Baudry (2008); cf. aussi Baudry (2017).

52. Lanfranchi (2015).

53. Bur (2018).

54. Etcheto (2012).

55. Ferriès (2007).

56. T. R. S. Broughton: The Magistrates of the Roman Republic, Atlanta, 1986 [1968] qui reconstitue les fastes des magistrats romains de la République.

57. Cosme (2007) reprend sous une forme synthétique une partie de ces recherches.

58. Virlouvet (1985), (1995), (2009).

59. Moatti (1997) prolongeait l'analyse jusqu'à proposer l'idée d'un essor de la rationalité qui aurait accompagné ce processus de construction d'une administration efficace et cohérente.

60. Moatti (1993) ; Demougin (1994); Moatti (1998).

61. Roddaz (1984).

62. Cf. Hurlet (1993), (1997). On attend aussi sur ce sujet le livre de Raphaëlle Laignoux, La fabrique de l'Empire sur les modes de mobilisation par les protagonistes des guerres civiles des ressources fondant leur légitimité.

63. Cf. Hurlet (2012b).

64. Briquel (2000).

65. Humm (2017a) et (2017b).

66. Humm (2005).

67. Humm (2012).

68. Baudry (2008).

69. Lanfranchi (2015).

70. Bastien (2007).

71. Berthelet (2015).

72. À titre d'exemple des travaux de cet auteur, voir l'article récent, Guilhembet (2016a). À propos de l'aristocratie, on attend le livre de Robinson Baudry sur les patriciens à la fin de la République.

73. Guilhembet (2016b).

74. Courrier (2014).

75. Cadiou (2018)

76. Bur (2018)

77. Le Doze (2014).

78. Akar (2013).

79. Jacotot (2013).

80. Moatti (2018).

81. David / Hurlet (à paraître).

82. Hölkeskamp (2008).

83. Benoist (2010).

84. Moatti (2009).

85. Voir notamment Hurlet (2012a) de façon circonstanciée.

86. Pour l'essentiel David (2006). 
87. Sans qu'elle soit toujours explicitement revendiquée, l'influence de Pierre Bourdieu reste forte.

88. Baudry / Hurlet (2016).

89. Itgenshorst / Le Doze (2017).

90. Laignoux (2014); Guilhembet / Laignoux / Montlahuc (à paraître).

91. Hurlet / Montlahuc (2018).

92. Augier / Baudry / Rohr Vio (à paraître).

93. Montlahuc (2019).

94. Après une première invitation à l'Institut de droit romain, Paris, en mars 1972. La conférence de Christian Meier a été publiée en 1973: "Clisthène et le problème politique de la polis grecque ", Revue internationale des Droits de l'Antiquité, $3^{\text {e }}$ série, tome XX, 1973, p. 115-159.

95. Bruhns / David / Nippel (1997).

INDEX

Mots-clés : République romaine, introduction

Schlüsselwörter : römische Republik, Einleitung

\section{AUTEURS}

\section{JEAN-MICHEL DAVID}

Jean-Michel David est professeur émérite d'histoire romaine à l'Université Paris 1 PanthéonSorbonne. Pour plus d'informations, voir la notice suivante.

\section{FRÉDÉRIC HURLET}

Frédéric Hurlet est professeur d'histoire romaine à l'Université Paris Nanterre. Pour plus d'informations, voir la notice suivante. 